\title{
The Contribution of Artificial Intelligence Tools in Screening for Cancer of the Cervix
}

\author{
Guesmi Lamia ${ }^{1,3}$, Nabli Lotfi ${ }^{2,4}$ and Bedoui Mohamed Hédi ${ }^{1}$ \\ 1. Laboratoire Technology and Medical Imaging, Faculty of Medicine, Ibn Sina Street 5019, Monastir, Tunisia \\ 2. Higher Institute of Applied Science and Technology of Sousse Box, Sousse 4000, Tunisia \\ 3. National Engineering School of Monastir, Route de Kairouan (ENIM), Monastir 5000, Tunisia \\ 4. Research Unit: Automatic, Signal Processing and Imaging (ATSI, ENIM), Monastir 5000, Tunisia
}

Received: April 06, 2011 / Accepted: May 04, 2011 / Published: June 25, 2011.

\begin{abstract}
Recently, research into pathological cytology were intended to put in places of artificial intelligence systems based on the development of new diagnostic technologies and the cell image segmentation. These technologies are not intended to substitute the human expert but to facilitate his task. The objective of this work is to develop a method for diagnosing cancer cervical smears using cervical-vaginal segmented to build the authors' database and a human supervisor and as an automatic tool manage and monitor the execution of the operation of diagnostic and proposing corrective actions if necessary. The Supervisor Smart is manufactured by the technique of neural networks with a success rate of $43.3 \%$ followed by the technique of fuzzy logic with a success rate equal to $56.7 \%$ and finally to improve this rate we used neuro-fuzzy approach which has a rate which reaches $94 \%$.
\end{abstract}

Key words: Cervical smear-vaginal (CSV), artificial intelligence, supervisor, fuzzy logic, neural networks.

\section{Introduction}

In Pathological Anatomy and Cytology, we distinguish two types of tests-the histology is the observation of the cutting of tissue and cytology is the examination of a spreading cell. We are particularly interested in cytology. The samples are spread on a slide and then fixed and stained to recognize the different cells present. The smears are then examined under a microscope by a cyto-technician to identify cells of interest. This step of reading the blade consists of a visual assessment of these cells onto a cytological blade. The purpose of this step is either the detection of abnormal or suspicious cells, namely the quantification of cells. This is of vital interest to the pathologist who must establish a reliable and valid

Nabli Lotfi, professor, research fields: automatic computer, integrated manufacturing and industrial data processing.

Bedoui Mohamed Hédi, professor, research field: technology and medical imaging.

Corresponding author: Guesmi Lamia, engineer, research field: automatically and plant maintenance. E-mail: lamia_guesmi@yahoo.fr. diagnosis especially in the case of classification CSVs for the diagnosis of cancer cervix. That is why we introduced artificial intelligence tools to facilitate this task with a very high success rate based on the technical supervisor of human and automatic after illustrating a priori information used to recognize cells are size, shape, texture but also and mainly the color [1-3]. The paper is organized as follows: Section 2 discusses the segmentation of CSV; section 3 introduces the shape parameters; section 4 discusses the methodologies of classification per supervisor of the vaginal smears cervico; section 5 discusses the application of the supervisor using the tools of artificial intelligence; section 6 presents simulation results; section 7 is discussion; section 8 gives conclusion; section 9 presents future work.

\section{Segmentation of CSV}

\subsection{Elimination of Inflammatory Cells}

In order to clean CSVs of inflammatory cells that 
invaded the image to the Treaty (Fig. 1), in which these cells recognize the same tent staining than nuclei of cells tested is why we spent at gray image to properly locate and illustrate the inflammatory cells using applications programming software MATLAB.

We turn to the gray level of the smear to the elimination of certain inflammatory cells that exhibit a level of RGB (red-green-blue) is not perfectly zero as the case of carcinogenic cell nucleus. We note that the cleaning of the smear is not clear that essentially means the occupation of certain sensitive places of inflammatory cells into the cell nucleus as a carcinogen or the borders of the cytoplasm of the cells in question, all this may give false decisions. And to avoid confusion, it is recommended to make a levy as sharp as possible by the cyto patologist, so do the cleaning cell by cell [4].

After this operation, we spent segmentation using smear, also operate in the programming software MATLAB.

\subsection{Image Segmentation in the Experimental Phase}

Segmentation plays a very important role in pattern recognition. It was after this stage that we can extract the parameters of quality will be defined with the continuation. Sometimes we have more than one cell in the image examined. Then we have to separate the whole cell from other cells that may appear on the edges of the image, that is to say, incomplete cells. Here are enumerated the steps required to segment such an image: Playing the picture; Detection internal and external contours of a cell linearization outlines of objects; Dilatation contours of objects filling internal objects; Removing objects exceeding the edge of the image, and the softening of the object and segmentation (outer contour only). We ended up following a CSV segmented (Fig. 2).

\section{Shape Parameters}

After segmentation of the image we had in carcinogenic cell sorting parameters to locate the most

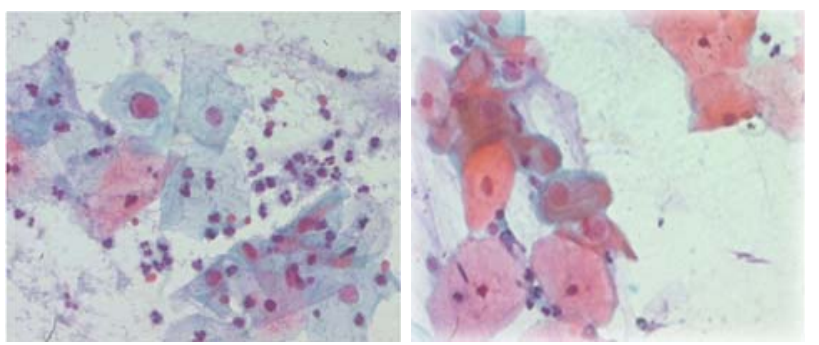

Fig. 1 Vaginal smear: the background is inflammatory and contains blue parasites. The squamous cells show clear perinuclear halo (Papanicolaou staining).
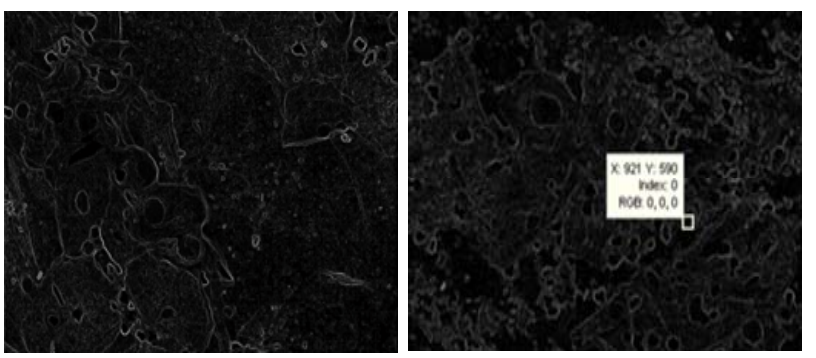

Fig. 2 Vaginal smear segmented: we can determine the overall shape of the cell carcinogenic and size of the nucleus and the cytoplasm (X,Y,index: punctual coordinates).

appropriate forms, each grade of cancer (class) is described by the parameters of quality based on the shape of the nucleus and cytoplasm more significant. They represent the best indicators used for the diagnosis and to achieve the classification of cancer. Their values are measured directly and manually to get them out. We have specified eight parameters of the form using the physician are

$$
\begin{gathered}
\mathrm{E} 1=\left(\mathrm{DF}_{\text {Max }}\right)_{\text {kernel }} /\left(\mathrm{DF}_{\mathrm{Max}}\right)_{\text {cytoplasm }} \\
\left.\mathrm{E} 2=(\mathrm{DF})_{\text {Min }}\right)_{\text {kernel }} /(\mathrm{DFMin})_{\text {cytoplasm }} \\
\mathrm{DF} 1=\left(\mathrm{DF}_{\mathrm{Min}} / \mathrm{DF}_{\mathrm{Max}}\right)_{\text {cytoplasm }} \\
\mathrm{DF} 2=\left(\mathrm{DF}_{\mathrm{Min}} / \mathrm{DF}_{\mathrm{Max}}\right)_{\text {kernel }} \\
\mathrm{U} 1=\mathrm{SN} 1 / \mathrm{SC} 1 \\
\mathrm{U} 2=\mathrm{SN} 2 / \mathrm{SC} 2 \\
\mathrm{SC} 1 / \mathrm{SC} 2
\end{gathered}
$$

$\mathrm{SN} 1 / \mathrm{SN} 2$

With $\mathrm{DF}_{\mathrm{Max}}$ : Maximum Feret diameter; $\mathrm{DF}_{\mathrm{Min}}$ : Minimal Feret diameter; SN1: Size of the smallest circle included the nucleus; SN2: Size of the largest circle circumscribing the nucleus SC1: Size of the smallest circle including the cytoplasm and SC2: Size of the largest circle circumscribing the cytoplasm. 


\section{Methodologies of Classification per Supervisor of the Vaginal Smears Cervico}

The establishment of a supervisor in the systems including human operators necessitates the implementation of intelligent interfaces [5-7]. This interface also adapts to changes in the human operator. It is necessary to take into account: its experience in operating the system, its physical condition and mental state especially for pathologists. All these criteria correspond to imperfect information modeled by fuzzy sets [6-9].

\subsection{Structure of Supervisor}

We will identify the famous approaches supervisor:

- Centralized: is characterized by controlling localized within a single resource that manages and oversees the production alone, in real time, events that occur throughout the production or development of the system [10];

- Decentralized: Its principle is to break a problem into sub-simpler problems of this type, to solve each of these sub-problems, then fusing the results to solve the problem [11];

- Hierarchical: This method accepts a control structure that stands at both the projected level (level 1) and real time (level 2). Each level coordinates the steering units of lower level, until the lowest level [12].

\subsection{Models of Supervisor}

There are several models which are to include the relationship between man as an operator and automation:

- Model Rasmussen: It is to model the activity monitoring accurately the decision-making process and insists on a knowledge representation of goal-directed [13-15];

- Model OCM (Optimal Control Model): The principle was extended to the detection of defects in a supervisory function of continuous variables of a multi-variable [16];
- Model for the regulation of human activity: It is not an order, but representation is meant to explain the regulation of human activity, for prediction of performance and workload and therefore to define the degree of automation (initial division of tasks between humans and automation) [17];

- Model of the technical system [18]: This type of model that is advanced compared to other models in which human roles are moving to supervisory responsibilities mostly making and much less reactive than previously [16];

- Model of human decision making [16]: It is characterized by a human operator built into the conduct or supervision of a large system is no longer viewed as merely reactive (optimal or not) but also as a solver problems.

4.3 Cooperation between the Automatic and the Human Operator: the Mixed Approach in the Structure of the Supervisor

There are two structures of cooperation between the automatic and the human operator:

- The structure of vertical cooperation: It has the operator as responsible for all process variables and may be called upon if necessary for the tool to the decision which will provide advice [17,19];

- Structure of horizontal cooperation: Dynamic Task Allocation: The outputs of the tool are connected to the actuators of the process control system, which means that this tool has capabilities for reasoning in real time [20].

We have selected as the model of human decision making is characterized by a human operator built into the conduct or oversight of a major system is no longer considered to be reactive (optimal or not) but also as a problem solver is the case of a doctor. And we have chosen as a structure is that of mixed vertical and horizontal cooperation which it presents a hand, the operator is to tell the doctor, is responsible for all process variables and he can call if necessary for the tool to the decision which will provide advice. 


\section{Application of the Supervisor Using the Tools of Artificial Intelligence}

In our case, we are interested diagnosis-based models and database of a hybrid system because our support, equal to $120 \mathrm{CSVs}$ distributed in equal shares to four classes of cancer (Cancer C), High Grade Dysplasia (HGD), Low Grade Dysplasia (LGD) and Normal $(\mathrm{N})$ ). These smears will be processed and exploited, in order to extract qualitative and quantitative information. These will form the basis of data for the development and testing of the tool to develop diagnostic techniques based on Artificial Intelligence (AI).

\subsection{Supervisor Achieved by the Technique of Neural} Networks (Multilayer Perceptron (MLP))

Neural networks have several types; the most used is the MLP which is characterized by its learning algorithm and back propagation of errors [21]. The MLP is an artificial neural network-oriented organized in layers, where information flows in one direction, from input layer to output layer. The input layer is always a virtual layer containing the entries of the system [22]. The following layers are the hidden layers that admit " $\mathrm{m}$ " number of layers according to the need for resolution of the system. It ends with the output layer representing the results of classification system [23]. In the case suggested, the MLP consists of an input layer which consists of eight shape parameters that are specified above, a hidden layer which consists of 26 neurons and an output layer is composed of four neurons formant the four main grades of cancer cervix. This type of perceptron is chosen after several tests, in which by varying the number of neurons in the hidden layer, the learning period and the vector normalization. We used the activity as a function of "Log-Sigmoid" with the normalization vector is $\mathrm{V}=[0.01,0.99]$, because the database is formed by values that are between 0 and 1 .

\subsection{Supervisor Achieved by the Technique of Fuzzy Logic}

The construction of a fuzzy model for the diagnosis and classification is to move mainly by three main stages: the fuzzification, the inference and deffuzzification. To achieve these three steps have to be set membership functions of input variables and those of output.

Definition of membership functions of input and output variables: The membership functions are most commonly used form: Singleton, Triangular, Trapezoidal, Gaussian. We retained the trapezoid for their simplicity of coding and manipulation, and it includes the range of variation of shape parameters. The number of trapezoidal shape in the representation of the membership function will be limited as much as possible for four to describe the scope of a variable. In this case they will be associated with terms: low-medium-large-very important.

We associate a variable $(\mathrm{Z})$ the following values (Table 1): minimum (Zmin), optimistic (ZTopt), optimistic (Zopt) average optimism (ZmoyO) average pessimistic (ZmoyP), pessimistic (Zpes), very pessimistic (Ztpes ) and maximum (Zmax).

The membership functions are shown in Figs. 3-11.

Fuzzification: After defining the membership functions according to the expert, we had to convert our input parameters for numerical values of physical quantities in translating linguistic variables and fuzzy since the algorithm needs exact numerical variables (1, $2,3,4,5, \ldots)$ to be usable by the following algorithm while the eight input parameters will admit four lexical classes: LOW, MEDIUM, IMPORTANT and VERY IMPORTANT where we have assigned their respective values ' 1 ', ' 2 ', ' 3 ' and ' 4 '. For the output vector allows four lexical classes "HGD", "LGD", "C" and "N" where we have respectively their associated values ' 1 ', '2', '3' and '4' [24].

Rules of inference or inference: This block is composed by all the fuzzy rules that exist between input variables and output variables expressed both as linguistic. We have chosen as a method of inference 
Table 1 Membership functions of input and output.

\begin{tabular}{lllllllll}
\hline & Zmin & ZTopt & Zopt & ZmoyO & ZmoyP & Zpes & Ztpes & Zmax \\
\hline E1 & 0.000 & 0.134 & 0.265 & 0.407 & 0.517 & 0.668 & 0.881 & 1.000 \\
E2 & 0.000 & 0.186 & 0.339 & 0.429 & 0.609 & 0.611 & 0.998 & 1.000 \\
DF1 & 0.000 & 0.383 & 0.391 & 0.484 & 0.833 & 0.936 & 0.960 & 1.000 \\
DF2 & 0.000 & 0.403 & 0.493 & 0.519 & 0.903 & 0.977 & 0.983 & 1.000 \\
U1 & 0.000 & 0.019 & 0.081 & 0.174 & 0.176 & 0.622 & 0.963 & 1.000 \\
U2 & 0.000 & 0.015 & 0.081 & 0.165 & 0.290 & 0.477 & 0.810 & 1.000 \\
SC1/SC2 & 0.000 & 0.154 & 0.174 & 0.210 & 0.754 & 0.802 & 0.913 & 1.000 \\
SN1/SN2 & 0.000 & 0.208 & 0.210 & 0.242 & 0.883 & 0.914 & 0.991 & 1.000 \\
S & 0.000 & 0.150 & 0.170 & 0.250 & 0.270 & 0.400 & 0.500 & 0.700 \\
\hline
\end{tabular}

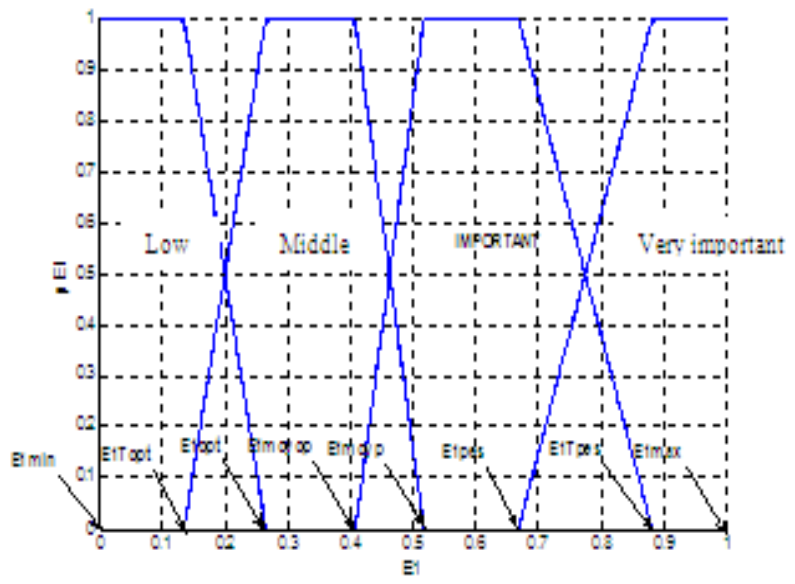

Fig. 3 Function of membership of $E 1$.

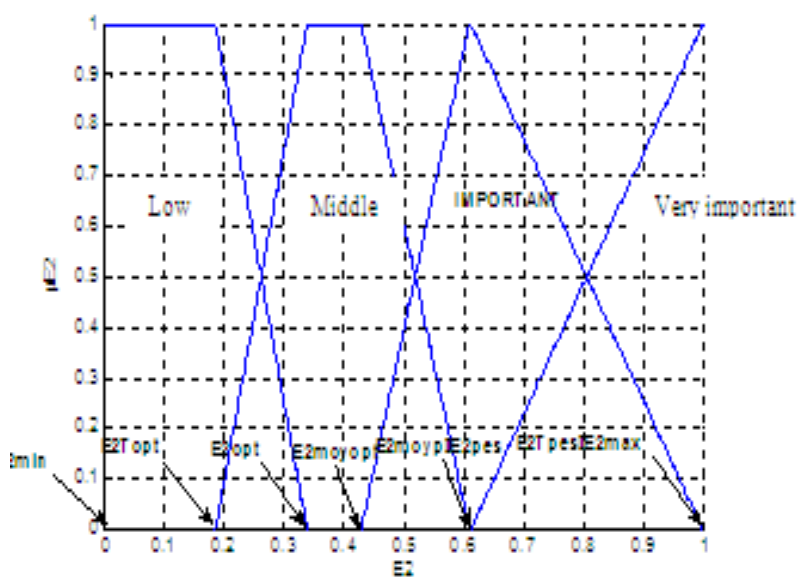

Fig. 4 Function of membership of $E 2$.

method "MAX-MIN". It is based on the use of two logical operations: the "AND" logic associated with the minimum and the "OR" logic associated to the maximum. These rules are of the form: IF (AND .......) THEN (decision) OR ... [25-26].

We have 185 possible rules of inference in the form

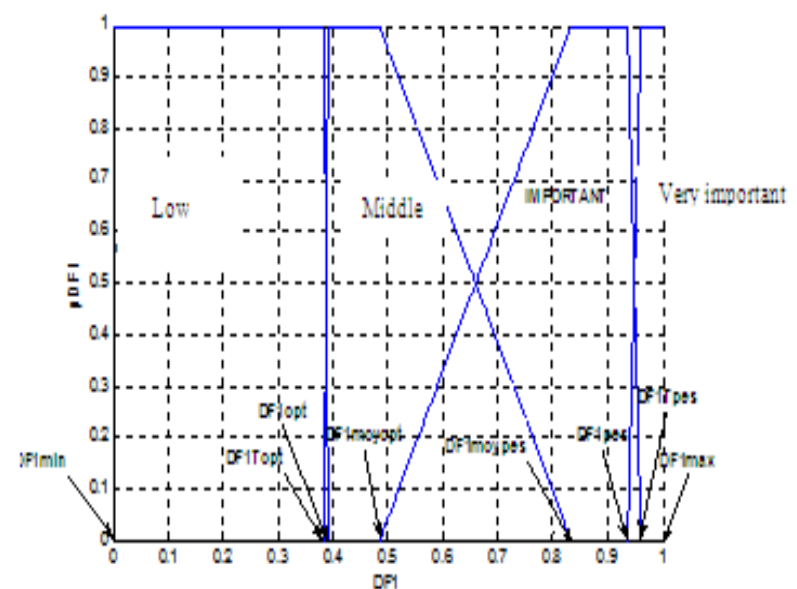

Fig. 5 Function of membership of DF1.

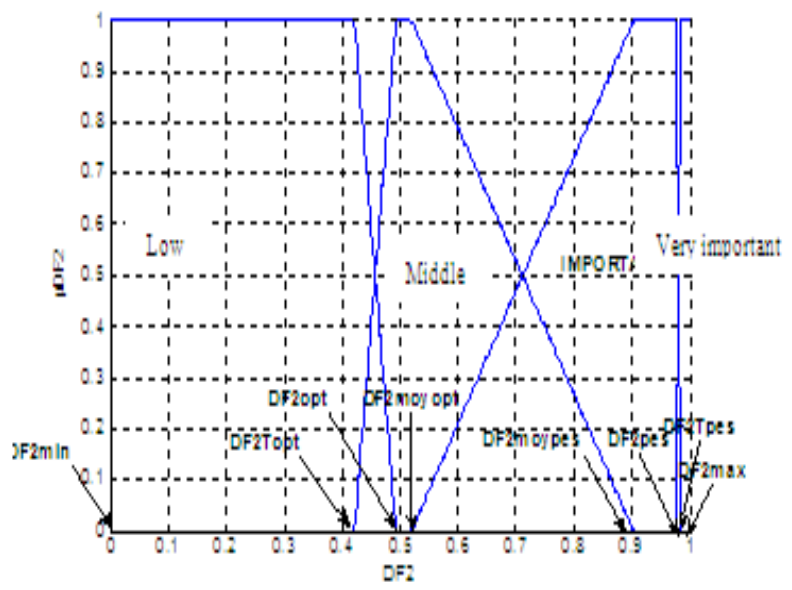

Fig. 6 Function of membership of $D F 2$.

of the maximum number of rules.

Defuzzification: The inference methods provide a membership function resulting " $\mu$ " for the output variable $x$. It is therefore fuzzy information that must be transformed into physical quantity. This conversion to reverse the phase of "fuzzification" [24-25]. 


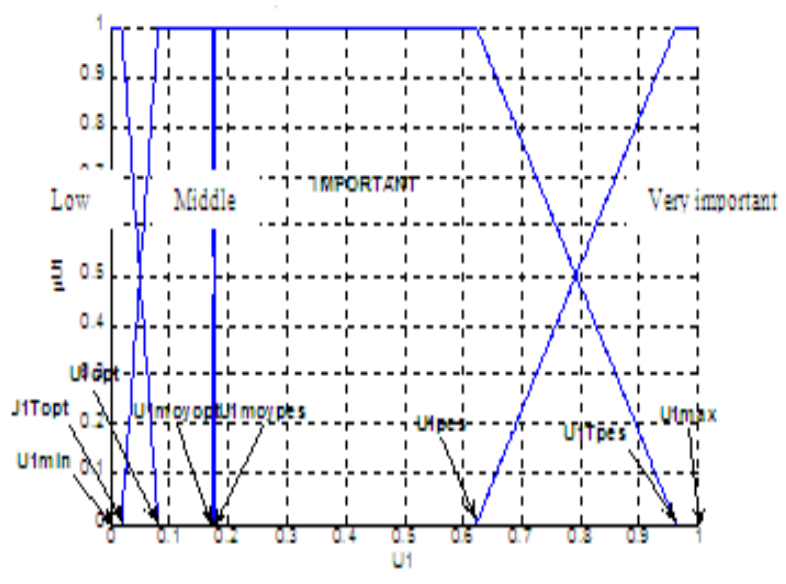

Fig. 7 Function of membership of $U 1$.

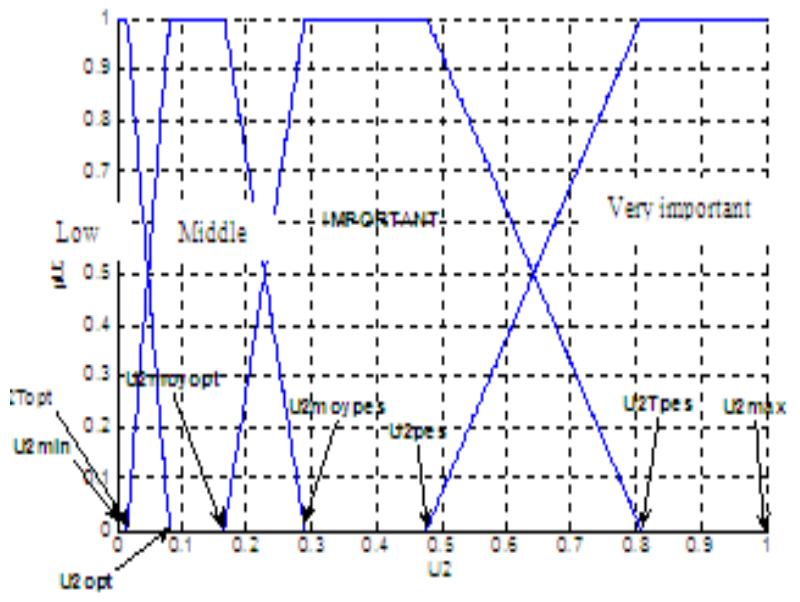

Fig. 8 Function of membership of $U 2$.

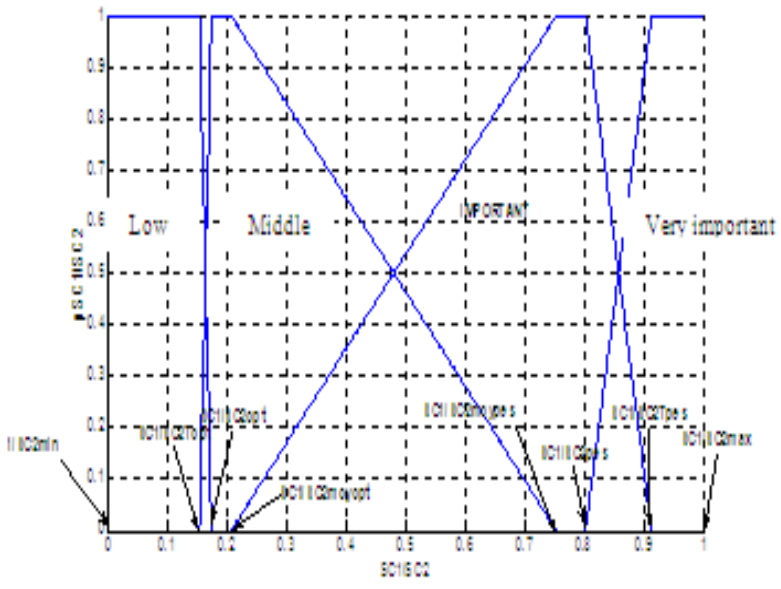

Fig. 9 Function of membership of SC1/SC2.

\subsection{Supervisor Directed by the Hybrid Approach: Neuro-Fuzzy}

Such a hybrid system is characterized by the following features: learning is performed by an

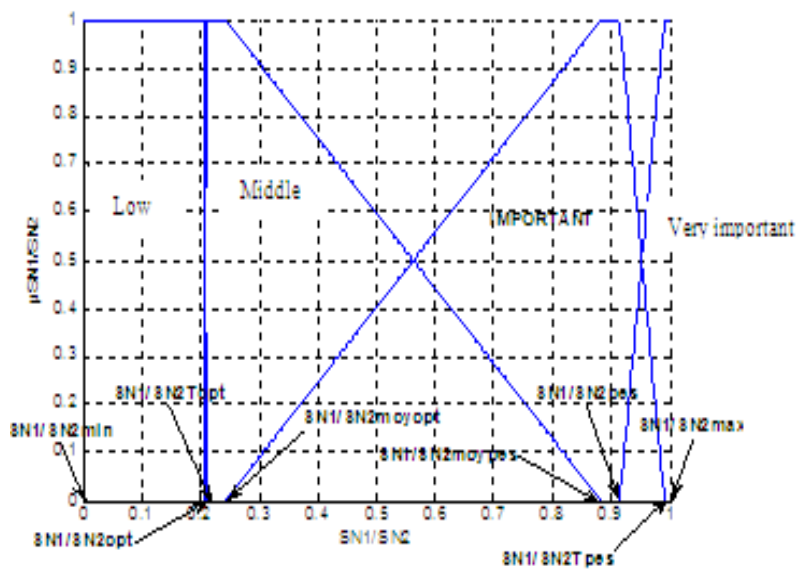

Fig. 10 Function of membership of SN1/SN2.

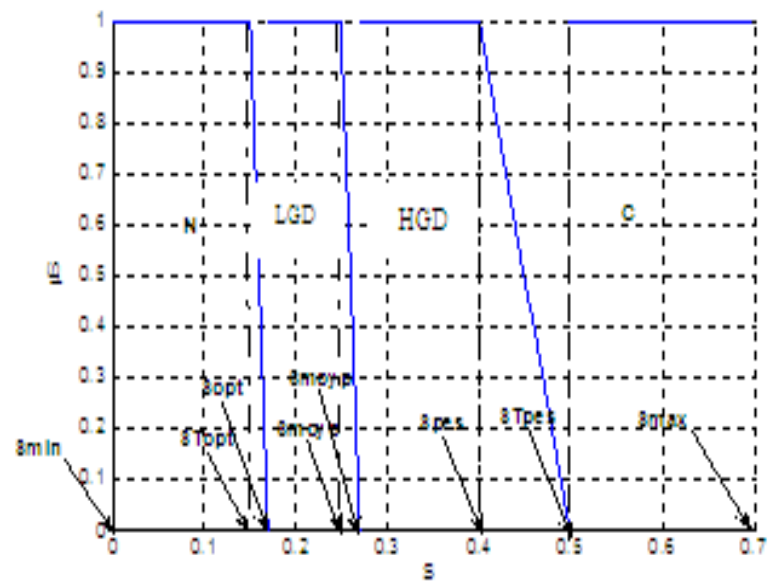

Fig. 11 Function of membership of $S$.

algorithm derived from a neural network, the general architecture of this system is represented by a recurrent network, this system is interpreted in terms of rules the form "if ... then ..." Learning is done from the semantics of the underlying fuzzy model, thus preserving the linguistic interpretability of the model, this model performs a function approximation. Therefore, the use of such an approach neuro-fuzzy overcomes the disadvantages inherent in each of the previous approaches (neural networks and fuzzy logic), while retaining their benefits [26-27].

\section{Simulation Results}

The results of the use of an intelligent supervisor in screening for cancer cervix in the three recurrent artificial intelligence techniques are recapitalized in the table (Table 2) after which it appears the success 
Table 2 The success rate of supervisors intelligent.

\begin{tabular}{llll}
\hline & $\begin{array}{l}\text { Neural } \\
\text { network (\%) }\end{array}$ & $\begin{array}{l}\text { Fuzzy } \\
(\%)\end{array}$ & $\begin{array}{l}\text { Neuro-fuzz } \\
\text { y (\%) }\end{array}$ \\
\hline HGD & 56.7 & 43.3 & 100 \\
LGD & 40 & 66.7 & 96.7 \\
$\mathrm{C}$ & 33.3 & 56.7 & 86.7 \\
$\mathrm{~N}$ & 43.3 & 60 & 93 \\
Total success rate (\%) & 43.3 & 56.7 & 94 \\
\hline
\end{tabular}

rate each technique for the same database (the 120 cases).

\section{Discussion}

The success rate achieved through the three approaches to artificial intelligence is very important for all the neuro-fuzzy case can be improved if we increase our database used. The present method neuro-fuzzy areas of confusion between two classes that are close to formal point of view of the cell is carcinogenic for couples (HGD, C) and (LGD, N). As a remedy, we can add another parameter form that expresses a specific quality indicator or as mentioned previously we grow our database. Despite these false positive cases, this method has the refuge of cancer screening cervical currently.

\section{Conclusions}

The supervisor directed by the hybrid approach neuro-fuzzy won the record classification CSVs a success rate of around $94 \%$ which we can improve if we increase our data down by other cases of injuries precancerous and cancerous encountered.

\section{References}

[1] Prof. Baillet, Cancérologie, Université Pierre et Marie Curie, 2004

[2] G. Body, P. Descamps, M. Jourdan, et al., Néoplasie intra épithéliale du col, EMC 597-A-10 60-200-A-10.

[3] M. Maalej, H. Rziga, F. Benna, Cancers du col de l'utérus, Cancérologie pratique, 1999.

[4] A. Smadja, H. Minh, Le col utérin normal, Revue Française des Laboratories 237 (1992) 37-40.

[5] D. Argent, Cancer du col de l'utérus, épidémiologie anatomie pathologique diagnostic évolution principes du traitement, dépistage, Rev. Prat. 49 (1999) 1923-1933.

[6] Groupement de Recherche en Automatisation Intégrée et
Systèmes Hommes-Machine, available online at: http://www.univ-valenciennes.fr/graisyhm/rapgt59900.doc

[7] GRAISyHM GT5: Méthodologie de Conception des Systèmes de Supervision, Développement d'une plateforme expérimentale dédiée à la supervision, Thème B12: distribution des traitements entre automatismes et opérateurs humains, available online at: http://www.univ-valenciennes.fr/graisyhm/rapgt59798

[8] M. Silveira, La distribution avec redondance partielle de modèles à événements discrets pour la supervision de procédés industriels, Thèse de l'Université Paul Sabatier de Toulouse. Laboratoire d'analyse et d'Architecture des Systèmes du CNRS, 2003, pp. 54-73.

[9] J.M. Hoc, Supervision et contrôle de processus, La cognition en situation dynamique, Presses Universitaires de Grenoble, 1996.

[10] D. Trenteseaux, Conception d'un système de pilotage distribué, supervisé et multicritère pour les systèmes automatisés de production, Thèse doctorat de l'institut National Polytechnique de Grenoble, 1996, pp. 16-35.

[11] A. Tajer, Contribution aux approches formelles de synthèse de commande spécifiée par grafcet, Thèse doctorat de l'université de Reims Champagne Ardenne, 2005, pp. 3-7.

[12] B. Gaudin, H. Marchand, Une approche modulaire pour le contrôle de systèmes à événements discrets concurrents, Université de RENNES 1 et INRIA Rennes. Equipe VerTeCs, IRISA, Campus université de Beaulieu, 2005, p. 21.

[13] G.D. d'Ayot, Coopération et évaluation cognitive d'agents artificiels pour la supervision, Thèse doctorat de l'institut National des sciences appliquées de Toulouse, 2005, pp. 21-27.

[14] C. Kolski, H. Ezzinedine, Conception et évaluation des IHM de supervision: éléments mésologiques, Université de Valenciennes et du Hainaut-Cambrésis, Laboratoire: LAMIH-UMR CNRS.

[15] J.M. Hoc, Supervision et contrôle de processus, La cognition en situation dynamique, Presses Universitaires de Grenoble, 1996.

[16] P. Millot, Systèmes Homme-Machine et Automatique, Université de Valenciennes et du Hainaut-Cambrésis, Laboratoire: LAMIH CNRS, Journées Doctorales d'Automatique JDA'99, Conférence Plénière, Nancy, septembre 1999, pp. 23-21.

[17] P. Millot, Supervision des procédés automatisés et ergonomie, Edition HERMES, Paris, Décembre 1988.

[18] M. Lind, Representing goals and functions of complex systems: an introduction to multilevel flow modelling, Technical Report 90-D-381 TU Denmark, 1990, pp. 7-11.

[19] B. Houriez, Acquisition des connaissances pour l'aide à la conduite et la supervision des procédés industriels, Habilitation à diriger les recherché, Université de 
Valenciennes, 1994, pp. 37-45.

[20] P. Millot, A. Kamoun, An implicit method for dynamic allocation between Man and computer in supervision posts of automated processes, in: 3rd IFAC Congress on Analysis Design and Evaluation of Man Machine Systems, Oulu, Finland, June, 1988, pp. 13-20.

[21] L. Buniet, Traitement automatique de la parole en milieu bruité: étude de modèles connexionnistes statiques et dynamiques, Thèse doctorat, Université Henri Poincaré-Nancy 1, Spécialité informatique, 1997.

[22] M.E. Zoghbi, Analyse électromagnétique et outils de modélisation couples, Application à la conception hybride de composants et modules hyperfréquences, Thèse doctorat, Université de Limoges, 2008.

[23] M.L. Hafiane, Conception d'un capteur de pression intelligent, Mastère en micro électrique, Option IC Design, Université de Batna: faculté des sciences de l'ingénieur, 2005.
[24] L. Nabli, Contribution à la Conduite des Systèmes de Production par l'utilisation des Techniques de l'Intelligence Artificielle, Habilitation Universitaire, Université de Monastir, 2010, pp. 100-104.

[25] G.O. CIMUCA, Système inertiel de stockage d'énergie associe à des générateurs éoliens, Thèse doctorat, Spécialité: Electrique, Ecole nationale supérieure d'arts et métiers centre de Lille, 2005, pp. 162-163.

[26] L. Baghli, Contribution à la commande de la machine asynchrone, utilisation de la logique floue, des réseaux de neurones et des algorithmes génétiques, Thèse doctorat, UFR Sciences et Techniques: STMIA, Université Henri Poincaré, Nancy-I, 1999.

[27] K.Främling, Les réseaux de neurones comme outils d'aide à la décision floue, Rapport de D.E.A., Spécialité: informatique. Equipe Ingénierie de l'Environnement, Ecole Nationale Supérieure des Mines de Saint-Etienne, 1992, pp. 11-12. 